DOC.

D101.60/4:

1112

Technical Report 1112

\title{
Effect of Viewing Conditions on Sickness and Distance Estimation in a Virtual Environment
}

Jennifer A. Ehrlich

University of Central Florida

Consortium Research Fellows Program

February 2000

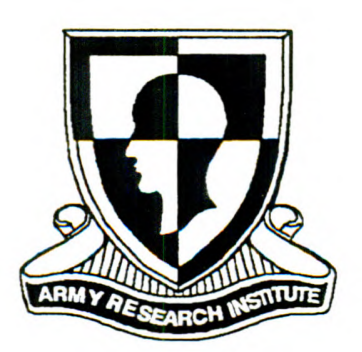

United States Army Research Institute for the Behavioral and Social Sciences

Approved for public release; distribution is unlimited. 